\title{
Relación entre el optimismo disposicional y los síntomas somáticos en pacientes en hemodiálisis crónica
}

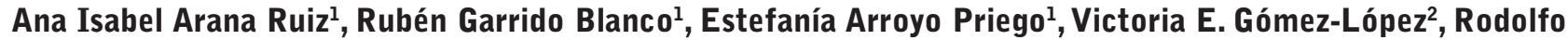 \\ Crespo Montero ${ }^{1,2,3}$ \\ ${ }^{1}$ Departamento de Enfermería. Facultad de Medicina y Enfermería. Universidad de Córdoba. España \\ 2 Unidad de Gestión Clínica de Nefrología. Hospital Universitario Reina Sofía de Córdoba. España \\ ${ }^{3}$ Instituto Maimónedes de Investigación Biomédica de Córdoba. España
}

\section{Resumen}

Objetivos: El objetivo del presente estudio fue analizar la relación existente entre el optimismo disposicional y el apoyo social, con los síntomas somáticos en un grupo de pacientes en hemodiálisis.

Pacientes y Método: Se estudiaron 80 pacientes en hemodiálisis, con una edad de $65,20 \pm 16,47$ años (36,3\% mujeres). Se realizó un estudio descriptivo y transversal, en el Servicio de Nefrología de Hospital Reina Sofía de Córdoba. Se estudió el Optimismo Disposicional (cuestionario LOT-R), el Apoyo Social (cuestionario Duke-UNC), los síntomas (Escala de Síntomas Somáticos Revisada) y la comorbilidad asociada.

Resultados: La media del LOT-R fue $21,19 \pm 5,04$ puntos y la mediana 21 (8-30). Se utilizó la mediana del LOT-R para dividir a los pacientes en Pesimistas Disposicionales $(<21)$ y Optimistas Disposicionales $(\geq 21)$. Aunque edad y comorbilidad fueron más altas en el grupo Optimista, las diferencias no fueron significativas. Los Optimistas presentaron menos síntomas que los Pesimistas, aunque sin diferencias significativas. Los pacientes con Apoyo Social más bajo presentaron más síntomas, aunque sin diferencias significativas. Tampoco se encontraron diferencias significativas en el Apoyo Social recibido entre Optimistas frente a los Pesimistas. Las mujeres presentaron más síntomas gastrointestina-

Correspondencia:

Ana Isabel Arana Ruiz

C/ Francisco José n 3. Esc. Dcha 7 B B4. 29003 Málaga

E-mail:Anabel_aruiz@hotmail.com les y músculoesqueléticos. La edad se correlacionó estadísticamente con la comorbilidad.

Conclusiones: Al menos en nuestra muestra, en los pacientes en hemodiálisis, ni el Optimismo Disposicional ni el Apoyo Social parecen influir en la aparición de síntomas somáticos, ni tampoco el Apoyo Social tiene influencia en el Optimismo Disposicional. Las mujeres presentan más síntomas gastrointestinales y músculoesqueléticos que los hombres. A más edad más comorbilidad asociada.

PALABRAS CLAVE: hemodiálisis; optimismo disposicional; síntomas somáticos; apoyo social.

\section{Relationship between dispositional optimism and somatic symptoms in patients on chronic hemodialysis}

\section{Abstract}

Objectives: The aim of the present study was to analyze the relationship between dispositional optimism and social support with somatic symptoms in a group of patients on hemodialysis.

Patients and Method: We studied 80 patients on hemodialysis, with an average age of $65.20 \pm 16.47$ years $(36.3 \%$ women and $63.7 \%$ men). A descriptive and cross-sectional study was carried out in the Nephrology Service of the Reina Sofía Hospital in Córdoba. The LOT-R questionnaire for Dispositional Optimism and the Revised Somatic Symptoms Scale were used. Social Support and associated comorbidity were also analyzed. 
Results: The mean LOT-R was $21.19 \pm 5.04$ and the median 21 (8-30). The median LOT-R was used to divide patients between pessimists $(<21)$ and optimists $(\geq 21)$. Although age and comorbidity were higher in the optimistic group, not being significant differences. Although optimists presented fewer symptoms than pessimists, there were no significant differences. Similarly, although patients with lower Social Support presented more symptoms, also without significant differences. When the Social Support of the optimists was compared with the pessimists, no differences were found. Statistical relationship of age was found with comorbidity and cardiovascular symptoms.

Conclusions: At least in our sample, in hemodialysis patients, neither Dispositional Optimism nor Social Support seem to influence the appearance of somatic symptoms, nor does Social Support influences Dispositional Optimism. Women have more gastrointestinal and musculoskeletal symptoms than men. The older, the more associated comorbidity.

KEYWORDS: hemodialysis; dispositional Optimism; somatic symptoms; social support.

\section{Introducción}

En España, existen alrededor de 57.000 pacientes con Enfermedad Renal Crónica Avanzada ${ }^{1}$ (ERCA) en Terapia Renal Sustitutiva (TRS), distribuyéndose de la siguiente manera: al 52,4\% de los mismos están con Trasplante Renal funcionante (TR), un $42,2 \%$ se encuentran en Hemodiálisis (HD) y un 5,5\% en Diálisis Peritoneal $\left(D^{2}\right)$. La ERCA afecta tanto a la esfera física como a la emocional, debido precisamente, a que precisa una TRS. La incorporación del paciente al tratamiento dialítico supone un desajuste importante en sus hábitos de vida, afectando a la calidad de la misma ${ }^{3}$.

La sintomatología derivada de la ERCA está bien definida, en base a las diferentes alteraciones que la misma conlleva en todos los sistemas o aparatos (renal, cardiocirculatorio, metabolismo óseo, etc). Sin embargo, muchos pacientes con adecuados niveles de hematocrito, un buen control bioquímico y correctamente dializados, siguen manteniendo molestias físicas que alteran su bienestar, para los cuales no se halla una etiología eviden$\mathrm{te}^{4}$, ni responden de manera óptima a los tratamientos individualizados prescritos 5 . La importancia otorgada al detrimento físico que sufre el enfermo renal puede con- trastarse con el gran desarrollo de la bibliografía que ha experimentado la calidad de vida relacionada con la salud $6,7,8$, que expone como los impedimentos físicos del paciente renal modifican de manera significativa las percepciones y valoraciones que efectúa de su condición y capacidad adaptativa. Sin embargo, son escasos los estudios que se hayan centrado en la prevalencia de los diferentes tipos de síntomas físicos en enfermos de $H D$, junto con la posible trascendencia de los componentes psicológico-emocionales en su determinación $n^{9}$. Todo esto puede llevarnos a una minusvaloración y un tratamiento insuficiente de esta sintomatología ${ }^{10}$.

Por otro lado, la capacidad del sujeto de controlar su propia conducta poniendo en práctica conductas de afrontamiento adaptativas va a ser crucial para una buena integración a todo el proceso de diálisis ${ }^{11}$. Este modelo de "autorregulación de conducta" sostiene que, cuando surgen adversidades, las expectativas favorables aumentan los esfuerzos de las personas para lograr sus objetivos promoviendo un afrontamiento activo y persistente; por el contrario, tener expectativas desfavorables reducen tales esfuerzos ${ }^{11,12}$.

Dentro de este modelo, el optimismo y el pesimismo serían considerados como expectativas generalizadas (favorables y desfavorables, respectivamente) acerca de las cosas que le acontecen a cada persona en la vida. Tales expectativas se consideran, además, como disposiciones estables, es decir, como rasgos; de aquí que Carver y Sheier (2001) señalen que el optimismo es disposicional11,13. Este planteamiento fue confirmado mediante los resultados de otros autores, donde el pesimismo se vinculó con la negación, distanciamiento de los eventos y una tendencia a centrarse en sentimientos estresantes; mientras que el optimismo disposicional (OD) se asoció con un enfoque centrado en el problema, la búsqueda de apoyo social y un énfasis en los aspectos positivos de la situación estresante ${ }^{14}$. Así, se ha comunicado que el optimismo se traduce en mayor calidad de vida y mejor bienestar físico y psicológico en algunas patologías crónicas ${ }^{15}$.

De otro lado, también se ha señalado la importancia del apoyo social (AS) en el afrontamiento de alteraciones crónicas, ayudando a realizar un afrontamiento más eficiente en circunstancias estresantes, disminuyendo la sensación de amenaza, los estados emocionales negativos y las respuestas orgánicas ante el estrés ${ }^{8,16}$. En enfermos renales se ha demostrado el impacto protector, mejorando varios aspectos relacionados con la enfermedad como pueden ser: la calidad de vida, la habitua- 
ción a la patología, la supervivencia, la percepción de la manifestación de síntomas relacionados con la problemática de salud mental y la adherencia al tratamiento ${ }^{17}$.

Por consiguiente, tanto el afrontamiento como el apoyo social ante esta enfermedad, podrían jugar un importante papel, en los desencadenantes de síntomas somáticos del paciente en HD y, por consiguiente, tener un efecto de la mejora de la salud y en la resistencia a la enfermedad. De ahí el interés de relacionar la sintomatología somática con el OD y AS, como posible explicación a la presencia de más síntomas físicos en pacientes con un tratamiento dialítico y farmacológico adecuado. Por todo ello, el objetivo del presente estudio fue analizar la relación existente entre el OD y el AS, con los síntomas somáticos en un grupo de pacientes en HD, mediante los siguientes objetivos específicos:

- Determinar la influencia del OD en la aparición de síntomas somáticos en pacientes en HD.

- Valorar el papel del AS en la aparición de síntomas somáticos en pacientes en HD.

\section{Material y Método}

\section{Ámbito de estudio}

El estudio tuvo lugar en la Unidad de Gestión Clínica de Nefrología del Hospital Universitario Reina Sofía (HURS), en la Unidad de Diálisis Hospitalaria (UDH), el Centro Periférico de Diálisis (CPD) y el Centro Periférico de Diálisis de Pozoblanco (CPDP) con la colaboración del personal de enfermería de estos centros.

\section{Diseño}

Se realizó un estudio observacional descriptivo y transversal.

\section{Población y muestra}

La población de estudio fueron todos los pacientes que se dializaban en los citados centros entre los meses de marzo-mayo. Se llevó a cabo un muestreo no probabilístico accidental de todos los pacientes que, tras leer la hoja informativa y el consentimiento informado, quisieron formar parte del estudio. Se excluyeron a aquellos pacientes con deterioro cognitivo.

De un total de 102 pacientes en programa de HD periódicas que tenía el programa en ese momento, 14 pacientes no quisieron participar, y 8 fueron excluidos por deterioro cognitivo.
La muestra estuvo formada por tanto por 80 pacientes (78\% del total), con una edad media de $65,20 \pm 16,47$ años. El $36,3 \%$ eran mujeres y el 63,8 fueron hombres. La recogida de datos se llevó a cabo durante las sesiones HD de los pacientes. Los cuestionarios fueron cumplimentados todos por una única entrevistadora para evitar sesgos de interpretación, garantizándose en todo momento la confidencialidad de los datos recogidos.

Se analizaron: el OD, el AS, los síntomas somáticos y la comorbilidad. Otras variables estudiadas fueron la edad, sexo, tiempo en diálisis y centro de HD.

\section{Instrumentos de medida}

Para evaluar el OD se utilizó el cuestionario validado LOT- $R$, el cual es una revisión del LOT, adaptado al castellano ${ }^{15}$.

Consta de 10 ítems en escala Likert de cinco puntos, donde 4 significa totalmente de acuerdo, 3 algo de acuerdo, 2 indiferente, 1 algo en desacuerdo, 0 totalmente en desacuerdo. Por un lado, seis de los ítems procuran medir la dimensión de OD, los cuatro ítems restantes son de "relleno" cuya función es hacer el cuestionario menos evidente. De los seis ítems de contenido, tres están redactados en sentido positivo u optimista y tres en sentido pesimista o negativo. A mayor puntuación obtenida, mayor será el grado de $0 D^{13,15}$.

Para medir el AS se utilizó el cuestionario Duke-UNC, que consta de 11 ítems y una escala de respuesta tipo Likert (1-5), variando el rango de puntuación entre 11 y 55 puntos (a menor puntuación menor apoyo). La puntuación alcanzada es un reflejo del apoyo percibido, no del real. En la validación española se optó por un punto de corte en el percentil 15, que corresponde a una puntuación de $<32$, es decir, una puntuación obtenida por debajo de 32 indica un apoyo social percibido bajo y por encima de 32 un apoyo social percibido normal ${ }^{18}$.

Finalmente, para medir la cantidad de síntomas se utilizó la Escala de Síntomas Somáticos Revisada (ESS-R). Contiene 90 ítems con formato de respuesta tipo Likert de 5 puntos, con un rango de 0 a 4; a mayor puntuación obtenida, más síntomas. Los síntomas se valoran en relación a la incidencia del último año y se organizan en subescalas: Inmunológico general, Cardiovascular, Respiratorio, Gastrointestinal, Neurosensorial, Piel-alergia, Musculoesquelético, Genitourinario y Reproductor femenino. Las dos últimas subescalas fueron excluidas del estudio. La genitourinaria está relacionada con la excreción de orina, lo cual está intrínsecamente alterado en los pacientes 
en HD; y la subescala del aparato reproductor femenino, solo es aplicable a mujeres fértiles ${ }^{10}$. Cada aparato o sistema consta de un número de preguntas concretas cuyo sumatorio es su resultado final.

Para medir la comorbilidad se utilizó el Índice de comorbilidad de Charlson.

\section{Aspectos éticos}

El estudio contó con el informe favorable del Comité de Ética e Investigación del HURS (Código TFGRODSS-2018).

\section{Análisis estadístico}

Para las variables cualitativas se utilizó una distribución de frecuencias, mientras que para las variables numéricas se usó la media \pm la desviación estándar de la media y en algunos casos la mediana. Para la comparación entre variables se utilizó la $t$ de Student para medias independientes. También se realizaron correlaciones con el test de Pearson. Se aceptó significación estadística para $p<0,05$. El análisis estadístico se ha realizado en un paquete estadístico SSPS 15.0 para Windows.

\section{Resultados}

Del total de la muestra, 39 pacientes $(48,8 \%)$ se dializaban en el CPD, $27(33,8 \%)$ en la UHD y $14(17,5 \%)$ lo hacían en el CPDP. El tiempo en HD medio fue de $104 \pm 111$ meses, y el ICC medio de la muestra fue $6,86 \pm 2,6$ puntos.

La media del LOT-R fue $21,19 \pm 5,04$ y la mediana 21 (rango:8-30). Se utilizó el valor de la mediana para dividir la muestra en dos grupos: en el primero se incluyeron los pacientes con un LOT-R $<21$, formado por 42 pacientes $(52,5 \%)$ al que denominamos Pesimistas Disposicionales (PD) y otro, formado por 37 pacientes $(47,5 \%)$, con una puntuación en el LOT-R $\geq 21$, a los que se denominó OD, para poder comparar a ambos grupos.

En la Tabla 1, se puede observar como los optimistas tenían más edad, tiempo en HD y comorbilidad que los pesimistas, aunque sin diferencias estadísticamente significativas. Respecto al AS obtenido con el cuestionario Duke-UNC, la media del total de la muestra fue $42,56 \pm 8,1$ puntos, si bien al clasificarlos, 8 pacientes ( $10 \%$ ) percibían un AS Bajo, y 72 (90\%) percibían un AS Normal.
Tabla 1. Comparación entre PD y OD para la edad, tiempo en HD e ICC.

\begin{tabular}{lccc}
\hline & PD & OD & Valor de p \\
\hline Edad & $62,3 \pm 18,5$ & $68,4 \pm 13,4$ & NS \\
Tiempo en HD & $98,7 \pm 117,1$ & $109,9 \pm 105,35$ & NS \\
ICC & $6,50 \pm 3,04$ & $7,26 \pm 2,11$ & NS \\
\hline
\end{tabular}

Edad: años. Tiempo en HD: meses. ICC: puntos. NS: No Significativo.

Al comparar el OD entre los pacientes que el AS Bajo frente a los que lo percibían Normal, no se encontraron diferencias significativas ( $19 \pm 3,5$ frente a $21,4 \pm 5,1 ; N S$ ).

Las puntuaciones medias y desviaciones típicas, de los síntomas somáticos (ESS-R) del total de la muestra, pueden observarse en la Tabla 2.

Tabla 2. Valores de la Escala de Síntomas Somáticos Revisada.

\begin{tabular}{lcc}
\hline Subescalas ESS-R & Media y DT & Min - Máx \\
\hline Inmunológico & $7,99 \pm 5,52$ & $00-21,00$ \\
\hline Cardiovascular & $10,41 \pm 6,11$ & $00-27,00$ \\
\hline Respiratorio & $8,54 \pm 7,29$ & $00-26,00$ \\
\hline Gastrointestinal & $7,05 \pm 6,29$ & $00-27,00$ \\
\hline Neurosensorial & $11,56 \pm 8,18$ & $00-35,00$ \\
\hline Músculo esquelético & $14,18 \pm 9,35$ & $00-38,00$ \\
\hline Piel y alergia & $6,01 \pm 5,24$ & $00-24,00$ \\
\hline Total síntomas & $65,74 \pm 33,51$ & $11,00-156,00$ \\
\hline
\end{tabular}

A mayor puntuación obtenida más síntomas, en relación a la incidencia del último año.

Al comparar los síntomas somáticos de los pacientes PD frente a los OD, podemos observar en la Tabla 3, como los pesimistas presentan en general, mayor valor para todas las subescalas del ESS-R que los optimistas, aunque sin diferencias estadísticamente significativas.

Tabla 3. Comparación los síntomas somáticos entre PD y OD.

\begin{tabular}{|lccc|}
\hline Subescalas ESS-R & PD & OD & Valor de $p$ \\
\hline Inmunológico & $8,62 \pm 5,17$ & $7,29 \pm 5,88$ & NS \\
\hline Cardiovascular & $11,00 \pm 6,41$ & $9,76 \pm 5,76$ & NS \\
\hline Respiratorio & $8,64 \pm 7,10$ & $8,42 \pm 7,60$ & NS \\
\hline Gastrointestinal & $7,64 \pm 6,16$ & $6,40 \pm 6,46$ & NS \\
\hline Neurosensorial & $11,60 \pm 7,07$ & $11,53 \pm 9,36$ & NS \\
\hline Musculo-esquelético & $14,02 \pm 9,35$ & $14,34 \pm 9,47$ & NS \\
\hline Piel y alergia & $5,64 \pm 4,55$ & $6,42 \pm 5,95$ & NS \\
\hline Total síntomas & $67,17 \pm 31,50$ & $64,16 \pm 35,97$ & NS \\
\hline
\end{tabular}

A mayor puntuación obtenida más síntomas, en relación a la incidencia del último año. 
Tampoco se encontraron diferencias estadísticamente significativas cuando se compararon los síntomas somáticos de los pacientes con AS Bajo frente a los que percibían un AS Normal, aunque los pacientes con AS Bajo puntuaron más alto en todas las Subescalas, excepto en la Cardiovascular, tal como puede apreciarse en la Tabla 4.

Con objeto de valorar la posible implicación de la comorbilidad en la aparición de síntomas somáticos, se utilizó la mediana del ICC (7 puntos), para hacer 2 grupos: pacientes con el ICC $\leq 7$ puntos y pacientes con el ICC $>7$ puntos, encontrándose diferencias significativas tan sólo entre la edad de ambos grupos, como puede apreciarse en la Tabla 5.

Cuando se analizó la influencia del sexo, en las distintas variables estudiadas, no se encontraron diferencias estadísticamente significativas en la edad, tiempo en HD, el LOT-R, el ICC y el AS, entre hombres y mujeres.

En cuanto a los síntomas somáticos, las mujeres presentaron más síntomas en las subescalas gastrointestinal y músculo-esquelético, que los hombres, siendo estas diferencias estadísticamente significativas, como puede apreciarse en la Tabla 6.
Tabla 4. Comparación los síntomas somáticos entre AS Bajo y AS Normal.

\begin{tabular}{|lccc|}
\hline Subescalas ESS-R & AS Bajo & AS Normal & Valor de $p$ \\
\hline Inmunológico & $11,13 \pm 6,98$ & $7,64 \pm 5,28$ & NS \\
\hline Cardiovascular & $9,50 \pm 5,78$ & $10,51 \pm 6,17$ & NS \\
\hline Respiratorio & $8,88 \pm 8,17$ & $8,50 \pm 7,25$ & NS \\
\hline Gastrointestinal & $9,50 \pm 5,78$ & $6,78 \pm 6,33$ & NS \\
\hline Neurosensorial & $11,75 \pm 9,85$ & $11,54 \pm 8,06$ & NS \\
\hline Músculo-esquelético & $15,88 \pm 10,20$ & $13,99 \pm 9,30$ & NS \\
\hline Piel y alergia & $6,38 \pm 5,15$ & $5,97 \pm 5,28$ & NS \\
\hline Total de síntomas & $73,00 \pm 37,25$ & $64,93 \pm 33,26$ & NS \\
\hline
\end{tabular}

A mayor puntuación obtenida más síntomas, en relación a la incidencia del último año.

Tabla 5. Comparación entre los pacientes con ICC $\leq 7$ puntos y $>7$ puntos.

\begin{tabular}{lccc}
\hline ICC & $\leq 7$ & $>7$ & Valor de $\mathbf{p}$ \\
\hline Edad & $59,2 \pm 17$ & $73,2 \pm 11$ & $\mathbf{P}<\mathbf{0 , 0 0 0 1}$ \\
LOT-R & $21 \pm 5$ & $21,4 \pm 5$ & NS \\
Síntomas (ESSR) & $65,2 \pm 35$ & $66,5 \pm 30$ & NS \\
AS & $43,6 \pm 8$ & $41 \pm 7$ & NS \\
\hline
\end{tabular}

Edad: años. OD, ESSR,AS: puntos. NS: No Significativo.

Tabla 6. Comparación los síntomas somáticos entre mujeres y hombres.

\begin{tabular}{|lccc|}
\hline Subescalas ESS-R & Mujer & Hombre & Valor de $\mathbf{p}$ \\
\hline Inmunológico & $8,51 \pm 4,86$ & $7,69 \pm 5,88$ & NS \\
\hline Cardiovascular & $10,10 \pm 6,02$ & $10,59 \pm 6,21$ & NS \\
\hline Respiratorio & $6,93 \pm 6,95$ & $9,45 \pm 7,39$ & NS \\
\hline Gastrointestinal & $9,10 \pm 7,08$ & $5,88 \pm 5,54$ & $\mathbf{P}<\mathbf{0 , 0 2 7}$ \\
\hline Neurosensorial & $13,83 \pm 7,86$ & $10,27 \pm 8,16$ & NS \\
\hline Musculo-esquelético & $17,97 \pm 10,07$ & $12,02 \pm 8,25$ & $\mathbf{P}<\mathbf{0 , 0 0 5}$ \\
\hline Piel y alergia & $5,79 \pm 5,62$ & $6,14 \pm 5,06$ & NS \\
\hline Total de síntomas & $72,24 \pm 33,87$ & $62,04 \pm 33,07$ & NS \\
\hline
\end{tabular}

A mayor puntuación obtenida más síntomas, en relación a la incidencia del último año.
La edad se correlacionó estadísticamente con el ICC $(r=0,586 ; p<0,001)$. No se encontraron más relaciones estadísticamente significativas.

\section{Discusión}

En el presente estudio hemos valorado la relación entre el OD y los síntomas somáticos y el AS en un grupo de pacientes en HD, dada su cronicidad, y la comorbilidad asociada que suelen tener estos pacientes. Para dividir a los pacientes en pesimistas y optimistas se utilizó la mediana como punto de corte, tal como han descrito otros autores ${ }^{11}$. Cuando se compararon las variables sociodemográficas de ambos grupos no se encontraron diferencias significativas, es decir, aparentemente ni el tiempo en HD, ni la edad, ni el ICC, parecen influir en el $O D$, algo que ya se ha reflejado en otros estudios ${ }^{19}$ y es coherente con el marco teórico de la capacidad de los sujetos de controlar su propia conducta poniendo en práctica conductas de afrontamiento adaptativas, independientemente incluso de la comorbilidad ${ }^{19}$.

Del mismo modo, al comparar los síntomas somáticos entre $P D$ y $0 D$, aunque la aparición de síntomas fue mayor en el grupo de pacientes pesimistas en todas las subescalas del ESS-R, estas diferencias, no fueron estadísticamente significativas, tal vez por el tamaño muestral. Sin embargo, algunos estudios han comunicado que ser optimista conlleva un afrontamiento activo de la 
enfermedad, lo que a su vez implica una disminución del dolor y de los síntomas ansioso-depresivos en pacientes con dolor crónico, y una mayor funcionalidad ${ }^{20}$; y en pacientes en $H D$, se ha sugerido que los pacientes con mejores puntuaciones en el $\mathrm{OD}$, presentan mejor percepción de calidad de vida ${ }^{11,19}$ y menos complicaciones asociadas al curso de la enfermedad renal ${ }^{11}$, frente al PD que desarrolla estrategias de afrontamiento pasivo ${ }^{6}$. Otros autores han comunicado que la variable OD está relacionada con una disminución de los síntomas de malestar físico ${ }^{21}$.

Con respecto al AS, no parece ser muy influyente en la muestra estudiada. En efecto, aunque la puntuación global de los síntomas somáticos de los pacientes con AS Bajo fue superior al de los pacientes con AS Normal, las diferencias no fueron estadísticamente significativas, si bien hay que destacar que tan sólo un 10\% de los pacientes tenían la percepción de un AS Bajo, por lo que la comparación estadística no es homogénea. Este resultado no concuerda con lo comunicado por otros autores, que encontraron una disminución de síntomas en pacientes con más $\mathrm{AS}^{10}$; en la misma línea que otros estudios que han mostrado que un buen nivel de AS se traduce en una mejora de la supervivencia y una reducción en cuanto a la percepción de la sintomatología $22-24$, y se relaciona de forma negativa con la morbi-mortalidad ${ }^{25}$. Tampoco hubo diferencias entre los pacientes con AS Bajo y AS Normal en la puntuación del OD.

Estos resultados sugieren que las variables psicológico-emocionales (depresión, grado de preocupación, estrategias de afrontamiento) pueden explicar algunos de los síntomas sin etiología clara en el enfermo renal, aunque no lo podemos confirmar, ya que no era objetivo del presente trabajo evaluar estas variables.

Respecto a la edad, se encontró una relación estadísticamente significativa entre esta y el ICC, es decir a mayor edad más comorbilidad; por lo que se decidió dividir a los pacientes en 2 grupo a partir de la mediana del ICC, encontrándose diferencias significativas exclusivamente en la variable edad, como era lógico, entre los pacientes que tenían un ICC $\leq 7$ puntos y los que tenían un ICC $>7$ puntos. No se encontraron diferencias para el LOT-T, síntomas somáticos y AS. Por tanto, tampoco parece explicar la comorbilidad la aparición de síntomas somáticos, que parecen estar más influidos por alteraciones emocionales como la ansiedad y la depresión ${ }^{10,26}$; aunque cuando se han estudiado los síntomas físicos de la ERCA tampoco se ha encontrado relación directa con la comorbilidad 27,28 .
En cuanto a la influencia del sexo en la aparición de síntomas somáticos, aunque la puntuación global de síntomas fue superior en las mujeres que en los hombres, estas diferencias no fueron significativas; excepto en las subescalas Gastrointestinal y Músculo-Esquelético, que las mujeres presentaron, de forma significativas, más síntomas que los hombres. No hemos encontrado otras referencias que confirmen este resultado, aunque si se ha descrito que las mujeres en HD presentan peor calidad de vida que los hombres ${ }^{29}$. Hay que reseñar, además, que el grupo de síntomas más recurrente en enfermos en diálisis son los músculo-esqueléticos, en comparación con otros grupos de síntomas 30,31 , posiblemente por la implicación dolorosa que conlleva ${ }^{32}$.

Como principal limitación de este estudio podemos destacar, que aunque la muestra no era pequeña, para este tipo de estudios, que se evalúan mediante cuestionarios con un componente subjetivo importante, es necesario analizar muestras más amplias para confirmar las tendencias, como la encontrada en nuestra muestra.

A la vista de estos resultados, podemos concluir que al menos en los pacientes en HD estudiados, el OD no tiene influencia en la aparición de síntomas somáticos. De igual forma, ni el AS ni la comorbilidad, parecen influir en la aparición de síntomas somáticos.

Las mujeres presentan mayor sintomatología gastrointestinal y músculo-esqueléticos que los hombres.

Finalmente, indicar que sería recomendable tener en cuenta las variables psicosociales, especialmente la ansiedad, a la hora de diseñar intervenciones de enfermería para mejorar la sintomatología somática de estos enfermos.

Recibido: 28 mayo 2018

Revisado: 1 junio 2018

Modificado: 22 junio 2018

Aceptado: 28 agosto 2018 


\section{Bibliografía}

1. Alcázar R, Orte L, Otero A. Enfermedad renal crónica avanzada. Nefrología. 2008;28(Supl 3):S3-6.

2. Informe de diálisis y trasplantes 2016. Registro de la Sociedad Española de Nefrología. [Consultado 15 Febrero 2018]. Disponible en: http://www.senefro. org/contents/webstructure/InformeREER_2016_ BURGOS.pdf

3. Sánchez CR, Rivadeneyra-Espinoza L, Aristil PM. Calidad de vida en pacientes bajo hemodiálisis en un hospital público de Puebla, México. AMC [internet]. 2016 [consultado 15 abril 2018]; 20(3):262-0 Disponible en: http://scielo.sld.cu/scielo.php?script=sci_arttext\&pid=S1025-02552016000300006

4. Álvarez-Ude F, Fernández-Reyes $M J$, Vázquez $A$, Mon C, Sánchez R, Rebollo P. Síntomas físicos y trastornos emocionales en pacientes en programa de hemodiálisis periódicas. Nefrología. 2001; 21(2):191-9.

5. Williams A, Manias E. A structured literature review of pain assessment and management of patients with chronic kidney disease. J Clin Nurs. 2008;17(1):69-81.

6. Perales-Montilla CM, García-León A, Reyes del Paso GA. Predictores psicosociales de la Calidad de Vida en pacientes con Insuficiencia Renal Crónica en tratamiento de hemodiálisis. Nefrología. 2012; 32(5):622-30.

7. Rodríguez M, Merino M, Castro M. Valoración psicométrica de los componentes físicos (CSF) y mentales (CSM) del SF-36 en pacientes insuficientes renales crónicos en tratamiento con hemodiálisis. Cienc Enferm. 2009;15(1):75-88.

8. Yarlas AS, White MK, Yang M, Saris-Baglam RN, Bech PG, Christensen T. Measuring the health status burden in hemodialysis patients using the SF-36 ${ }^{\circledR}$ health survey. Qual Life Res. 2011;20(3):383-9.

9. Weisbord SD, Shields AM, Mor MK, Sevick MA, Homer M, Peternel $\mathrm{J}$ et al. Methodology of a randomized clinical trial of symptom management strategies in patients receiving chronic hemodialysis: The SMILE study. Contemp Clin Trials. 2010; 31(5):491-7.
10. Perales-Montilla CM, Duschek S, Reyes-del Paso $\mathrm{GA}$. Influencia de los factores emocionales sobre el informe de síntomas somáticos en pacientes en hemodiálisis crónica: relevancia de la ansiedad. Nefrología. 2013;33(6):816-25.

11. Morales-García AI, Arenas-Jiménez MD, Reig-Ferrer A, Álvarez-Ude F, Malek T, Moledous A et al. Optimismo disposicional en pacientes en hemodiálisis y su influencia en el curso de la enfermedad. Nefrología. 2011;31(2):199-05.

12. Chico-Librán E. Optimismo disposicional como predictor de estrategias de afrontamiento. Psicothema. 2002; 14(3):544-50.

13. Ferrando PJ, Chico E, y Tous JM. Propiedades psicométricas del test de optimismo Life Orientation Test. Psicothema. 2002;14(3):673-80.

14. Scheier MF, Weintraub JK, Carver CS. Coping with stress: divergent strategies of optimists and pessimists. J Pers Soc Psychol. 1986; 51(6):1257-4.

15. Rondón JE, Angelucci LT. Análisis psicométrico del Life Orientation Test (LOT-R) en población diabética venezolana. SEAS. 2016;22(1):11-8.

16. Uchino BN, Cacioppo JT, Kiecolt-Glaser JK. The relationship between social support and physiological processes: a review with emphasis on underlying mechanisms and implications for health. Psychol Bull. 1996;119(3):488-531.

17. Cukor D, Cohen SD, Peterson RA, Kimmel PL. Psychosocial aspects of chronic disease: ESRD as a paradigmatic illness. J Am Soc Nephrol. 2007; 18(12):3042-55.

18. Bellón JA, Delgado A, Luna J, Lardelli P. Validez y fiabilidad del cuestionario de apoyo social funcional Duke-UNC-11. Aten Primaria. 1996;18(4):153-63.

19. Luque I, López-Toledano MC, Torollo I, Crespo R. Evaluación del optimismo disposicional en pacientes en hemodiálisis y su relación con las complicaciones. Enferm Nefrol. 2014;17(4):261-8.

20. Ramírez-Maestre C, Esteve R, López AE. The role of optimism and pessimism in chronic pain patients adjustment. Span J Psychol. 2012;15(1):286-4. 
21. Remor E, Amorós M, Carrobles JA. El optimismo y la experiencia de ira en relación con el malestar físico. Anal Psicología. 2006;22(1):37-44.

22. Kimmel PL. Psychosocial factors in dialysis patients. Kidney Int. 2001;59(4):1599-13.

23. Uchino BN. Social support and health: a review of physiological processes potentially underlying links to disease outcomes. J Behav Med.2006;29(4):37787.

24. Patel SS, Peterson RA, Kimmel PL: The impact of social support on end-stage renal disease. Semin Dial. 2005;18(2):98-02.

25. Gil-Cunquero JM, Marrón B. La realidad y la percepción de las infecciones en diálisis. Nefrología. 2010;1(1):56-62.

26. Perales-Montilla CM, Duschekb S, Reyes del Paso G. Calidad de vida relacionada con la salud en la enfermedad renal crónica: relevancia predictiva del estado de ánimo y la sintomatología somática. Nefrología. 2016;36(3):275-82.

27. Gutiérrez-Sánchez $\quad D$, Leiva-Santos JP, Macías-López MJ, Cuesta-Vargas AI. Perfil sintomático de los pacientes con Enfermedad Renal Crónica Estadio 4 y 5. Enferm Nefrol. 2017;20(3):259-66.
28. Son Y-J, Choi K-S, Park Y-R, Bae J-S, Lee J-B. Depression, symptoms and the quality of life in patients on hemodialysis for end-stage renal disease. Am J Nephrol. 2009;29(1):36-42.

29. Albañil T, Ramírez-Moreno MC, Crespo R. Análisis de la calidad de vida en pacientes en hemodiálisis ambulatoria y su relación con el nivel de dependencia. Enferm Nefrol. 2014;17(3):167-74.

30. González C, Pizarro C. Dolor crónico en hemodializados: prevalencia, severidad y estrategias de afrontamiento. El dolor. 2009;51:19-25.

31. Calls J, Rodríguez MA, Hernández D, Gutiérrez MJ, Amer FJ, Tura D et al. Evaluación del dolor en hemodiálisis mediante diversas escalas de medición validadas. Nefrología. 2009;29(3):236-43.

32. Gómez-Pozo M, Ruiz-Parrado MC, Crespo-Garrido M, Gómez-López VE, Crespo-Montero R. Caracterización del dolor en el paciente en hemodiálisis. Enferm Nefrol. 2017;20(4):295-04.

Este artículo se distribuye bajo una Licencia Creative Commons Atribución-NoComercial 4.0 Internacional. https://creativecommons.org/licenses/by-nc/4.0/ 